Castro, H.O.; Costa, G.C.; Lage, G.M.; Praça, G.M.; Fernández-Echeverría, C.; Arroyo, M.P.; Greco, P.J. (2019) Visual Behaviour and Decision-Making in Attack Situations in Volleyball. Revista Internacional de Medicina y Ciencias de la Actividad Física y el Deporte, vol. 19 (75) pp. 565-578 Http://cdeporte.rediris.es/revista/revista75/artcomportamiento1040.htm

\title{
ORIGINAL
}

\section{COMPORTAMIENTO VISUAL Y TOMA DE DECISIONES EN SITUACIONES DE ATAQUE EN VOLEIBOL}

\section{VISUAL BEHAVIOUR AND DECISION-MAKING IN ATTACK SITUATIONS IN VOLLEYBALL}

\author{
Castro, H.O.1; Costa, G.C. ${ }^{2}$; Lage, G.M.3; Praça, G.M.4; Fernández- \\ Echeverría, C. ${ }^{5}$ Moreno, M.P. ${ }^{6}$ y Greco, P.J. ${ }^{7}$
}

1 Coordinador y profesor, Centro Universitário Estácio Brasília (Brasil) henriquecastro88@yahoo.com.br

2 Profesor, Universidade Federal de Goiás (Brasil) conti02@hotmail.com

3 Profesor, Universidade Federal de Minas Gerais (Brasil) menezeslage@gmail.com

4 Profesor, Universidade Federal de Minas Gerais (Brasil) gibson moreira@yahoo.com.br

5 Estudiante de doctorado, Facultad de Ciencias del Deporte, Universidad de Extremadura (España) cafernandeze@unex.es

6 Profesora, Facultad de Ciencias del Deporte, Universidad de Extremadura (España) pmoreno@unex.es

7 Profesor, Universidade Federal de Minas Gerais (Brasil) pablojgreco@gmail.com

Código UNESCO / UNESCO code: 5312.99 Deporte profesional / Profesional sports

Classificación Consejo de Europa / Council of Europe classification: 17. Otras: Entrenamiento deportivo / Others: Sports training

Recibido 3 de septiembre de 2017 Received September 3, 2017

Aceptado 25 de octubre de 2017 Accepted October 25, 2017

\section{RESUMEN}

El objetivo de estudio fue comparar el comportamento visual (número y duración de las fijaciones visuales) y la toma de decisiones (TD) en el análisis de escenas de ataque en voleibol, entre entrenadores y jóvenes jugadores. Participaron 59 sujetos - 34 entrenadores $(M=32.5 ; D T=9.4)$ con experiencia como jugadores y entrenadores de voleibol $(M=16.78$; $D T=11.09)$, y 25 jugadores $(M=16.9 ; D T=1)$ con experiencia como jugadores de voleibol $(\mathrm{M}=3.72$; $\mathrm{DT}=1.17)$. Para el análisis de la calidad de la TD se emplearon las escenas de ataque del Test de Conocimiento Táctico Declarativo en Voleibol (TCTD:Vb). Para el análisis del comportamiento visual se utilizó el Eye Tracking SMI RED500® durante el 
análisis de las escenas. Los resultados muestran diferencias significativas en la duración de las fijaciones siendo mayores en el grupo de los entrenadores. Concluye que existe un patrón similar de comportamiento visual em la comparación entre los grupos unicamente diferenciado por la duración de las fijaciones visuales.

PALABRAS CLAVE: Comportamiento visual; Toma de decisión; Voleibol.

\begin{abstract}
The objective of comparing visual behavior (number and duration of visual fixations) and decision-making (DM) in the analysis of attack scenes in volleyball, between coaches and young players. 59 subjects will participate -34 coaches $(M=16.7, S D=9.4)$ with experience as players and volleyball coaches $(M=16.78, S D=11.09)$, and 25 players $(M=16.9, S D=1)$ with experience as volleyball players $(\mathrm{M}=3.72, \mathrm{SD}=1.17)$. For the analysis of $\mathrm{DM}$ quality, the attack scenes of the Tactical Declarative Knowledge Test in Volleyball (TDKT:Vb) were used. For the analysis of visual behavior, the Eye Tracking SMI RED500® was used during the analysis of the scenes. The results show significant differences in duration of fixations being greater for the coaches group. It concludes that there is a similar pattern of visual behavior in the comparison between groups only differentiated by the duration of the visual fixations.
\end{abstract}

KEY WORDS: Visual behaviour; Decision-Making; Volleyball.

\title{
INTRODUCCIÓN
}

En voleibol, como en otros juegos deportivos, los jugadores se relacionan con el ambiente por medio de sus sentidos. Así, el jugador recibe, fundamentalmente via sistema visual, informaciones que, de acuerdo con su experiencia, se relacionan con su conocimiento táctico, para confluir en la toma de decisiones TD (Kreitz, Furley, Memmert, \& Simons, 2014). Las informaciones que surgen del ambiente para concretar una solución (mental y motora) del problema de juego, revelan la necesidad de centrar la atención visual en señales relevantes, señales que proporcionen pistas que faciliten la consecución de los objetivos de la acción y de la TD (Carrasco, 2011).

La TD emerge influenciada por factores como el número de alternativas para la solución de las situaciones de juego, la compatibilidad de estímulo-respuesta (Sanfey, 2007), la cantidad de práctica (Gil, Moreno, García-González, Moreno, \& Villar, 2012; Gil, Moreno, Moreno, García-González, Claver, \& Del Villar, 2013), la presión de tiempo (Raab, 2015), los factores cognitivos como la percepción (Janelle \& Hatfield, 2008), el nivel de atención (Hüttermann \& Memmert, 2014; 2015), las condiciones y experiencia para la anticipación (Tenenbaum, 2003), la memoria, fundamentalmente de la interacción entre la memoria de trabajo y la memoria a largo plazo (Furley \& Memmert, 2012; 2015), de la elaboración del 
pensamiento y de la inteligencia táctica (Afonso, Garganta, \& Mesquita, 2012). Además de esto, los jugadores expertos saben como y cuándo utilizar su mayor nivel de conocimiento (Arroyo, Domínguez, Arias, García-González, \& Álvarez, 2016), y su experiencia, en relación a los noveles, identificando y manipulando de forma eficiente la información relevante en cualquier momento, y permitiendo una TD más rápida y exitosa (Castro, Praça, Costa, Pedrosa, \& Greco, 2016; Oliveira, Lobinger, \& Raab, 2014; Raab \& Laborde, 2011; Araújo, Afonso, \& Mesquita, 2011).

Los estudios realizados con entrenadores han incidido fundamentalmente em conocer cuáles son las concepciones que éstos tienen sobre las metodologías de enseñanza (Schempp, Webster, McCullick, Busch, \& Mason 2007), sobre su autopercepción de competencia (Egerland, Nascimento, \& Both, 2010) y sobre su formación profesional (Brasil, Ramos, \& Nascimento, 2015). La bibliografia relacionada con la formación de entrenadores valora cada vez más el desarrollo de los procesos reflexivos sobre los problemas de la práctica (Jones, Morgan, \& Harris, 2012; Paquette, Hussain, Trudel, \& Camiré, 2014), que emerge de forma situada, en particular sobre la forma en la que los entrenadores transforman su conocimiento y competencias en decisiones concretas (Gilbert, Gilbert, \& Trudel, 2001). Sin embargo, no se han encontrado en la bibliografía revisada, estudios que analicen la calidad de la TD de los entrenadores en relación con los problemas emergentes del juego.

Una forma de investigar sobre este tema puede ser mediante el análisis del comportamiento visual medido a través del movimiento ocular con el instrumento denominado "Eye Tracking". El Eye Tracking se aplica con éxito en el estudio de una amplia variedad de fenómenos relacionados con el comportamiento visual (por ejemplo, atención y percepción) en diversas áreas de conocimiento, entre las que se encuentra el área de las ciências del deporte (DUCHOWSKI, 2007). En estudios de atención visual y percepción en el deporte, por ejemplo, el movimiento ocular es utilizado para investigar la focalización de la atención en el contenido de la imagen, la identificación de objetos y cómo las decisiones son tomadas (Castro, Praça, Costa, Pedrosa, \& Greco, 2016; Larsson, Nyström, Andersson, \& Stridh, 2015; Araújo, Afonso, \& Mesquita, 2011).

Conocer el funcionamiento de los procesos decisionales contribuye a mejorar el rendimiento de los desportistas expertos y a optimizar la formación de los principiantes (Vila-Maldonado, Abellán, Saéz-Gallego, García-López, \& Contreras, 2014). Sin embargo, Raab y Harwood (2015) indican que todavía hay lagunas en el conocimiento sobre los procesos que impulsan el desarrollo de la pericia en el deporte y fuera del deporte. De acuerdo con Schlappi-Lienhard y Hossner (2015), a pesar de que varios estudios examinaron la TD relacionada con la percepción visual y conocimiento específico de la modalidad deportiva, el estado actual de la investigación muestra limitaciones relativas a: (1) análisis de la percepción visual con los sistemas de movimiento ocular en entrenadores de voleibol, (2) identificación de aspectos de juego relevantes para el conocimiento de domínio específico, (3) interacciones entre ambos aspectos para la TD de entrenadores y deportistas en la modalidad de voleibol. 
Teniendo en cuenta que un mayor conocimiento especifico de un deporte se encuentra relacionado con una mejor TD, el presente estudio tiene como objetivo comparar el comportamiento visual (número y duración de las fijaciones visuales) y la TD de entrenadores y jóvenes jugadores de voleibol, en el análisis de escenas de ataque.

\section{MATERIAL Y MÉTODOS}

\section{Cuidados éticos}

La investigación fue aprobada por el Comité de Ética en Estudios de la Universidad Federal de Minas Gerais - COEP/UFMG (Parecer 821.295), y todos los voluntarios, así como sus representantes legales, concedieron el consentimento escrito para la participación en el estudio.

\section{Muestra}

Participaron en el estudio 59 sujetos, de ellos, 34 eran entrenadores de voleibol $(M=32.5$; $D T=9.4)$ con experiencia como entrenadores de voleibol $(M=16.78$; $\mathrm{DT}=11.09)$, y 25 eran jugadores de voleibol $(M=16.9$; $\mathrm{DT}=1)$ con experiencia como jugadores de voleibol $(M=3.72 ; D T=1.17)$. Dichos sujetos fueron selecionados de manera no probabilística intencional, conformándose como una muestra por conveniencia.

El critério de inclusión para la participación en el estudio se basó en que los voluntarios no presentasen ningún tipo de ceguera, estrabismo o cualquier otro problema de visión que pudiese comprometer la realización del estudio, además de estar regularmente inscritos en la Federación Mineira de Voleibol (FMV) y/o Confederación Brasileña de Voleibol (CBV) y disputaran competiciones con su respectivo club a nivel estatal, nacional y/o internacional. Todos los voluntarios que no cumplieron cualquiera de estos criterios fueron automáticamente excluidos del estudio.

\section{Variables de estudio}

Las variables consideradas en el estudio fueron de dos tipos: comportamiento visual, y toma de decisiones.

\section{Comportamiento visual}

- Número de fijaciones visuales: se refiere al número de veces que el sujeto fijó la mirada en un punto por un período de tiempo $\geq 100 \mathrm{~ms}$ (Sáez-Gallego, VilaMaldonado, Hernández, \& Jordán, 2013; Afonso, Garganta, McRobert, Williams, \& Mesquita, 2012; Afonso, Garganta, McRobert, Williams, \& Mesquita, 2014; Vila-Maldonado, Abellán, Saéz-Gallego, García-López, \& Contreras, 2014). 
- Duración de las fijaciones visuales: se refiere a la duración media de las fijaciones realizadas por cada sujeto, medidas en milisegundos (Sáez-Gallego, Vila-Maldonado, Hernández, \& Jordán, 2013).

\section{Toma de Decisiones (TD)}

- Calidad de TD: esta variable se basa en la cantidad de aciertos obtenidos por cada voluntario en las diferentes escenas presentadas (Sáez-Gallego, VilaMaldonado, Hernández, \& Jordán, 2013; Vila-Maldonado, Abellán, SaézGallego, García-López, \& Contreras, 2014). La cantidad de respuestas correctas (buena calidad de TD) tuvo en cuenta las respuestas de los expertos a la misma acción realizada por el jugador en la escena y culminaran en punto (garantizando la validez ecológica de la escena).

\section{Instrumentos}

\section{Test de Conocimiento Táctico Declarativo en Voleibol (TCTD:Vb)}

Para valorar la calidad de la TD se utilizaron escenas de ataque del Test de Conocimiento Táctico Declarativo en Voleibol - TCTD:Vb (Costa, Castro, Cabral, Morales, \& Greco, 2016). Las situaciones de ataque del test consisten en 12 escenas reales de juegos de voleibol pasadas en un ordenador. Dichas escenas fueron presentadas en dos tipos de situaciones: Ataque en Ala - AA (seis escenas) y Ataque de Centro - AC (seis escenas). Las escenas presentadas tenian uma duración de cuatro a seis segundos y fueron filmadas desde um optimo plano de vision (distancia de siete a nueve metros de la cancha de juego y una altura de 1,5 metros), permitiendo al observador la visión total de la cancha y la percepción de profundidad en las diferentes situaciones.

Las situaciones de juego que debían ser observadas por los sujetos, de AA y AC se iniciaban a partir del saque contrario, recogían la recepción del equipo del otro lado de la red, y la colocación, y se interrumpía la imagen en el momento del ataque.

Durante la realización del test, en el momento de interrupción de la escena, la pantalla se apaga y el sujeto tiene tres segundos para responder "¿qué hacer?" de forma verbal, de acuerdo con la respuesta que considere más adecuada (Liu, 2015). En este momento, el investigador responsable anota manualmente, en una ficha de control de recogida de datos, la respuesta del sujeto para cada momento y situaciones presentadas. Se utilizó ese tiempo debido a la conclusión de las acciones de juego en voleibol, que duran aproximadamente tres segundos. De este modo, se analizó la precisión de la respuesta como la TD del sujeto de acuerdo con las respuestas ya descritas en el instrumento (Castro, Praça, Costa, Pedrosa, \& Greco, 2016). 


\section{Eye Tracking}

Para el análisis del comportamiento visual (número y duración de las fijaciones visuales) durante la aplicación del test, fue utilizado el Eye Tracking SMI RED500®, de la empresa SensoMotoric Instruments - SMI. El Eye Tracking consta de un conjunto de tecnologías utilizadas para la identificación y registro de los movimientos oculares de un individuo en un contexto real o controlado (Sáez-Gallego, Vila-Maldonado, Hernández, \& Jordán, 2013). La base de desarrollo de la herramienta de análisis de la visión sigue los princípios metodológicos y prácticos sobre el comportamiento de la trayectoria ocular (Tien, Pucher, Sodergren, Sriskandarajah, Yang, \& Darzi, 2014; Duchowski, 2007).

Su aplicabilidad se sustenta por el hecho de ser un método seguro y no invasivo capaz de medir movimientos oculares precisos, no fácilmente accesibles por otro método (DUCHOWSKI, 2007), además de la íntima relación entre la atención y el comportamiento ocular en tareas visuales (como en el caso de los deportes de colaboración-oposición) (NUMMENMAA; HYÖNÄ; CALVO, 2006). La mayoría de los estudios que han utilizado este instrumento lo han hecho con estímulos estáticos, como imágenes de fotos y textos, pero actualmente el interés en escenas dinámicas (como el uso de videoclips) va creciendo cada vez más entre los investigadores (Larsson, Nyström, Andersson, \& Stridh, 2015).

Con este instrumento, el movimiento de los ojos de los sujetos se graba por un sistema de laser infrarrojo fijo y la pantalla de un ordenador (Tien, Pucher, Sodergren, Sriskandarajah, Yang, \& Darzi, 2014; Hansen \& Hammoud, 2007). El Eye Tracking ofrece datos referentes a variables relacionadas con el comportamiento visual (Vila-Maldonado, Abellán, Saéz-Gallego, García-López, \& Contreras, 2014), utilizando en este estudio el número y la duración de las fijaciones visuales (Castro, Praça, Costa, Pedrosa, \& Greco, 2016; Tien, Pucher, Sodergren, Sriskandarajah, Yang, \& Darzi, 2014).

Inicialmente, antes de comenzar con el test, el equipamiento se calibra a la altura y distancia correspondiente a los ojos del voluntario. Para ello, se utiliza el software iView X RED $500 \mathrm{~Hz}$, dicho software está incluido en el programa y es el recomendado por el fabricante. El test para la grabación de los movimientos oculares se realiza gracias al software Experiment Center 3.5, donde se programan las figuras y escenas de los videos, creándose así, el protocolo de la investigación presentada. Seguidamente, se procede a la fijación del cuello del voluntario por medio de un collarin hinchable que evita que el sujeto lo mueva. Después del grabado del movimiento ocular del sujeto por el equipamiento anteriormente presentado, los datos se archivan automáticamente en la memoria del ordenador y se transportan al software BeGaze 3.5.7.4, con el cual, es posible abrir el archivo de cada sujeto y cada variable en cada escena de video. Las variables relacionadas con el comportamiento visual son el número y duración de las fijaciones visuales. 


\section{Procedimiento de recogida de datos}

La recogida de datos se realizó en las propias canchas de entrenamiento y en el laboratorio de la Universidad. Previamente, se contactó con los participantes para conocer su disponibilidad de horario y así concretar en la agenda una fecha exacta para la prueba y recolección de datos. En el día marcado para la toma de datos, los sujetos fueron conducidos hasta una sala apropriada para la realización de los procedimentos de estudio y completaron el cuestionario de datos demográficos.

Seguidamente, las escenas de vídeo de TDTD: Vb fueron presentadas a los sujetos seguiendo el protocolo definido por el processo de aporte de instruciones relativas a la realización del test. Antes de comenzar con el test, los sujetos fueron sometidos a una calibración del equipamento y familiarización con los procedimientos que iban a ser realizados. Esa familiarización consistió en visualizar dos escenas de cada situación (AA y AC) con duraciones de cuatro a seis segundos, siguiendo exactamente los mismos protocolos de la situación experimental. Terminada la adaptación, sin ninguna pregunta o duda, los sujetos iniciaban los test experimentales cuando deseaban.

Los voluntarios realizaron el TCTD:Vb utilizándose el Eye Tracking en el momento de proyección de las escenas. Con este instrumento, se analizó el comportamiento visual (número y duración de fijaciones visuales, que hace referencia a la percepción de los puntos en los que el sujeto visualizó como señales relevantes), y durante la escena el sujeto expresó declarativamente su TD (Tien, Pucher, Sodergren, Sriskandarajah, Yang, \& Darzi, 2014; SáezGallego, Vila-Maldonado, Hernández, \& Jordán, 2013; Afonso, Garganta, McRobert, Williams, \& Mesquita, 2012; Afonso, Garganta, McRobert, Williams, \& Mesquita, 2014; Vila-Maldonado, Abellán, Saéz-Gallego, García-López, \& Contreras, 2014).

\section{Análisis Estadístico}

El análisis descriptivo se realizó mediante la media y la desviación típica (DT). Se realizaron las pruebas de normalidad (test de Shapiro-Wilk), homogeneidad de varianza (test de Levene) y esfericidad (test de Mauchly), los cuales no presentaron valores significativos. Seguidamente, se realizó un $t$ test para muestras independientes, recogiendo el poder del efecto (Phi), manteniéndose un nivel de signifitividad de $5 \%$. Todos los análisis fueron realizados en el software SPSS 20.0.

\section{RESULTADOS}

La tabla 1 presenta los resultados del análisis de las escenas de AA del TCTD: $\mathrm{Vb}$ en la comparación de entrenadores y jóvenes jugadores. 
Tabla 1. Comparación de lo comportamiento visual entre entrenadores y jóvenes jugadores en las escenas de AA del TCTD: $\mathrm{Vb}$

\begin{tabular}{cccccccc}
\hline \multicolumn{2}{c}{ Entrenador x Jugador } & $\mathrm{N}$ & Media & $\mathrm{DT}$ & $\mathrm{t}$ & $\mathrm{P}$ & $\mathrm{Phi}$ \\
\hline $\begin{array}{c}\text { Número de } \\
\text { fijaciones en } \\
\text { AA }\end{array}$ & $\begin{array}{c}\text { Entrenador } \\
\text { Jugador }\end{array}$ & 36 & 81.69 & 13.59 & & & \\
\hline $\begin{array}{c}\text { Duración de } \\
\text { las fijaciones } \\
\text { en AA }\end{array}$ & $\begin{array}{c}\text { Entrenador } \\
\text { Jugador }\end{array}$ & 36 & 24.08 & 20.07 & -0.32 & 0.17 & 0.68 \\
\hline
\end{tabular}

*diferencia encontrada para $\mathrm{p}<0.05$

$\mathrm{AA}=$ Ataque de Ala; $\mathrm{DT}=$ Desviación Típica; $\mathrm{Phi}=$ Poder del efecto

Se observa en la tabla 1 que el tiempo de fijación de los entrenadores fue significativamente mayor que el de los jóvenes jugadores, en el análisis de las escenas de AA.

La tabla 2 presenta los resultados relativos al TCTD: Vb realizado con las escenas de AC.

Tabla 2. Comparación de lo comportamiento visual entre entrenadores y jóvenes jugadores en las escenas de AC del TCTD: Vb

\begin{tabular}{cccccccc}
\hline \multicolumn{2}{c}{ Entrenador x Jugador } & $\mathrm{N}$ & Media & $\mathrm{DT}$ & $\mathrm{T}$ & $\mathrm{P}$ & $\mathrm{Phi}$ \\
\hline $\begin{array}{c}\text { Número } \\
\text { de }\end{array}$ & $\begin{array}{c}\text { Entrenador } \\
\text { Jugador }\end{array}$ & 36 & 81.69 & 13.59 & & & \\
$\begin{array}{c}\text { fijaciones } \\
\text { en AC }\end{array}$ & 24 & 83.08 & 20.07 & -0.32 & 0.17 & 0.70 \\
\hline $\begin{array}{c}\text { Duración } \\
\text { de las }\end{array}$ & $\begin{array}{c}\text { Entrenador } \\
\text { Jugador }\end{array}$ & 36 & $27893^{*}$ & 3105.16 & & & \\
$\begin{array}{c}\text { fijaciones } \\
\text { en AC }\end{array}$ & 24 & 20640 & 7203.65 & 5.36 & $<0.001$ & 1.00 \\
\hline
\end{tabular}

$\mathrm{AC}=$ Ataque de Centro; DT = Desviación Típica; $\mathrm{Phi}=$ Poder del efecto

Se observa en la tabla 2 que el tiempo de fijación de los entrenadores fue mayor que el tiempo de fijación de los jugadores, en el análisis de las escenas de AC.

\section{DISCUSIÓN}

El presente estudio comparó el comportamiento visual (número y duración de las fijaciones visuales) y la toma de decisión de entrenadores y jóvenes jugadores de voleibol. Hasta el momento, no se han encontrado estudios que comparasen entrenadores con jugadores en las variables toma de decisión y comportamiento visual, por tanto, serán presentados y discutidos resultados de investigaciones que estudiaron essas variables separadamente con atletas de distintas experiências en la modalidade, haja visto que los entrenadores y jugadores del presente estúdio se diferencian em esto aspecto. 
Los estudios sobre las estrategias visuales realizados hasta el momento con desportistas muestran que, el tiempo de práctica o la categoría en la que los desportistas están entrenando y compitiendo, es uno fator que diferencia estos comportamientos visuales (Crespi, Robino, Silva, \& De'Sperati, 2012; Afonso, Garganta, McRobert, Williams, \& Mesquita, 2012; Afonso, Garganta, McRobert, Williams, \& Mesquita, 2014). Al analizar a los entrenadores, podemos pensar que, probablemente ellos transportaron, a la situación actual, sus experiencias y conocimientos de la época en la que fueron desportistas. Así, el tiempo de práctica deliberada en el presente estudio evidenció una diferencia en los procesos relativos a la duración de las fijaciones, pero no en el número de las fijaciones y toma de decisión.

De acuerdo con los estudios en el área de conocimiento táctico (Gorman, Abernethy, \& Farrow, 2011; Abernethy, Baker, \& Côte, 2005) es posible afirmar que existe transferencia del conocimiento entre situaciones que comparten características semejantes organizacionales, estruturales y tácticas. Sin embargo, la interpretación de las diferentes situaciones requiere la relación entre mecanismos sensoriales (Banks \& Krajicek, 1991) y mecanismos de memoria (Stokes, Atherton, Patai, \& Nobre, 2012).

En los estudios realizados por Afonso y Mesquita (2013) y Afonso, Garganta, McRobert, Williams y Mesquita (2012) con jugadoras de voleibol divididas en dos grupos: habilidosas ( $N=9 ; M=16.1$; $D T=2.0$ años de edad) y menos habilidosas $(\mathrm{N}=6 ; \quad M=16.8 ; \quad \mathrm{DT}=2.0$ años de edad), utilizándose el análisis del comportamiento visual y los relatos verbales en escenas dinámicas de las tareas ofensivas, con el fin de tener una mayor comprensión de los mecanismos que hay detrás de la TD en el deporte. Los resultados de este estudio mostraron diferencias significativas en la duración media de las fijaciones, encontrándose que las jugadoras más habilidosas realizaron fijaciones más duraderas en comparación con las jugadoras menos habilidosas. En las variables número de fijaciones y número de lugar de fijaciones no fueron encontradas diferencias significativas entre los grupos, lo que está de acuerdo com los resultados encontrados en el presente estudio, en la comparación del grupo con más experiencia (entrenadores) con el de menos experiencia (jugadores).

En otro estudio, Piras, Lobietti y Squatrito (2010) encontraron que los jugadores de voleibol expertos, en comparación con los principiantes, realizaron un menor número de fijaciones de mayor duración en las manos y en el cuerpo del contrario, principalmente en el colocador, buscando alguna información para prever la trayectoria del balón, lo que también se corrobora con el presente estudio.

Estudios realizados por Castro, Praça, Costa, Pedrosa y Greco (2016), Liu (2015) y Piras, Lobietti y Squatrito (2014), con jugadoras de voleibol, demuestran que los expertos realizan fijaciones más rápidas, en comparación con los noveles. Estos resultados no corroboran los encontrados en el presente estudio y pueden ser explicados porque los expertos tuvieran mayor conocimiento específico de la modalidad, y hicieran más uso del tiempo para sus informaciones disponibles, fijando la atención durante más tiempo en las tareas. Esto permite 
que codifiquen y recuperen informaciones de forma eficiente y tomen decisiones más adecuadas.

Los resultados de presente estudio mostraron diferencias significativas en la experiencia con el voleibol cuando comparamos los grupos (entrenadores $\mathrm{x}$ jugadores), siendo mayor en el caso de los entrenadores. También se observaron diferencias significativas cuando comparamos los tiempos de fijación entre los dos grupos, siendo mayor en el caso de los entrenadores. Se hace explícito aquí la importância de dirigir el entrenamiento a focalizar la atención en los lugares específicos que demuestren uma mejor calidad en las informaciones de señales relevantes, con el objetivo de realizar una toma de decisión más eficaz.

En relación a la TD, no se encontraron diferencias en la comparación entre los grupos. De acuerdo con Schlappi-Lienhard y Hossner (2015) la TD depende de los detalles de información emitida en situación de juego por el contrario, el contexto externo, el contexto situacional específico y los movimentos del contrario. Además, existe una alta influencia del comportamiento visual y del dominio del conocimiento específico de la modalidade. Es importante que la transferência de aprendizaje considere la influencia de experiencias anteriores sobre la realización de una acción en un nuevo contexto.

Araújo, Afonso y Mesquita (2011) demonstraron que jugadores con mejores desempeños competitivos presentan mayores valores porcentuales de decisiones correctas, cuando son comparados con jugadores con peores desempeños competitivos en diferentes categorías de voleibol. Resultados éstos que no fueron corroborados en el presente estudio, en el cual no se apreciaron diferencias significativas en TD cuando se compararon los grupos de entrenadores y jugadores.

Resultados similares a los del presente estudio fueron encontrados en los trabajos de Vila-Maldonado, Abellán, Saéz-Gallego, García-López y Contreras (2014) y Araújo, Neves y Mesquita (2012), con jugadores de voleibol de diferentes categorías y experiencias específicas en la modalidad.

En el presente estudio, los resultados similares encontrados en la comparación entre los grupos utilizados (entrenadores y jóvenes jugadores) puede ser explicado por el hecho de que todos los entrenadores han sido exjugadores y el nivel competitivo de los jugadores de la muestra sea alto, lo que incluye competiciones a nivel nacional e internacional. Con esto, las diversas situaciones de juego vivenciadas por los dos grupos, a pesar de la diferencia en el tiempo de práctica de la modalidad, probablemente haya influido para que no haya diferencia en las calidades de la toma de decisión. En presente estúdio, ambos los grupos (entrenadores y jugadores) tienen critérios similares a la hora de decidir, y um nivel de toma de decisiones similar, y además, tambien presentan um perfil de comportamiento visual similar em cuanto al número de fijaciones, centrándose en similar numero de aspectos, pero dedicando un tiempo diferente a los mismos. Esto podría explicarse o relacionarse con el papel que realizan ambos normalmente, y con el hecho de que los jugadores estén acostumbrados 
a responder em mayor déficit de tiempo, ajustándose a las situaciones concretas de juego, cuestión que no es igual en el caso de los entrenadores.

\section{CONCLUSIONES}

Se observa que la duración de las fijaciones visuales fue mayor en el grupo de entrenadores, cuando se comparo con el grupo de jóvenes jugadores, pero esta situación no influyó en la calidad de TD, ya que no hubo diferencias significativas. Los resultados obtenidos permiten concluir que el tiempo empleado en analizar y codificar los estímulos provenientes del ambiente (señales relevantes), es diferente entre entrenadores y jugadores de voleibol.

Se sugiere, para estudios futuros, el análisis del comportamiento visual comparando diferentes categorías y grupos de jugadores y entrenadores, con distintas experiencias prácticas y competitivas en la modalidad de voleibol, con el fin de verificar cuál de los factores tiene mayor influencia para una toma de decisión eficaz.

\section{REFERENCIAS BIBLIOGRÁFICAS}

Abernethy, B.; Baker, J. \& Côté, J. (2005). Transfer of pattern recall skills may contribute to the development of sport expertise. Applied Cognitive Psychology, vol. 19, pp. 705-718. doi: 10.1002/acp.1102

Afonso, J.; Garganta, J.; McRobert, A.; Williams, A.M. \& Mesquita, I. (2012). The perceptual cognitive processes underspinning skilled performance in volleyball: Evidence from eye-movements and verbal reports of thinking involving an in situ representative task. Journal of Sports Science and Medicine, vol. 11, pp. 339345.

Afonso, J.; Garganta, J.; McRobert, A.; Williams, A.M. \& Mesquita, I. (2014). Visual search behaviours and verbal reports during film-based and in situ representative tasks in volleyball. European Journal of Sports Science, vol. 14 (2), pp. 1-8. doi: 10.1080/17461391.2012.730064

Afonso, J.; Garganta, J. \& Mesquita, I. (2012). A tomada de decisão no desporto: o papel da atenção, da antecipação e da memória. Revista Brasileira de Cineantropometria e Desempenho Humano, vol. 14 (5), pp. 592-601. doi: 10.5007/1980-0037.2012v14n5p592

Afonso, J. \& Mesquita, I. (2013). Skill-based differences in visual search behaviours and verbal reports in a representative film-based task in volleyball. International Journal of Performance Analysis in Sport, vol. 13, pp. 669-677. doi: 10.1080/24748668.2013.11868679

Araujo, R.; Afonso, J. \& Mesquita, I. (2011). Procedural knowledge, decisionmaking and game performance analysis in Female Volleyball's attack according to the player's experience and competitive success. International Journal of Performance Analysis in Sport, vol. 11, pp. 1-13. doi: 10.1080/24748668.2011.11868524

Araujo, R.M.F.; Neves, J.A. \& Mesquita, I.M.R. (2012). Procedural knowledge, decision-making and performance in women's volleyball according to age group 
and specific experience. The Open Sports Science Journal, vol. 5, pp. 167-173. doi: 10.2174/1875399X01205010167

Banks, W.P. \& Krajicek, D. (1991). Perception. Annual Review of Psychology, vol. 42, pp. 305-331. doi: 10.1146/annurev.ps.42.020191.001513

Brasil, V.Z.; Ramos, V. \& Nascimento, J.V. (2015). Propostas conceituais a respeito do conhecimento profissional do treinador esportivo de jovens. Revista da Educação Física, vol. 26 (3), pp. 483-493. doi: 10.4025/reveducfis.v26i3.24225

Carrasco, M. (2011). Visual attention: The past 25 years. Vision Research, vol. 51 (13), pp. 1484-1525. doi: 10.1016/j.visres.2011.04.012

Castro, H.O.; Praça, G.M.; Costa, G.C.T.; Pedrosa, G.F. \& Greco, P.J. (2016). Visual behaviour and quality of decision-making on volleyball. Revista Brasileira de Cineantropometria e Desempenho Humano, vol. 18(6), pp. 638-647. doi: 10.5007/1980-0037.2016v18n6p638

Costa, G.C.T.; Castro, H.O.; Cabral, F.A.; Morales, J.C.P. \& Greco, P.J. (2016). Content validity of scenes of the Declarative Tactical Knowledge Test in Volleyball - DTKT:Vb. Revista Brasileira de Cineantropometria e Desempenho Humano, vol.18 (6), pp. 629-637. doi: 10.5007/1980-0037.2016v18n6p629

Crespi, S.; Robino, C.; Silva, O. \& De'Sperati, C. (2012). Spotting expertise in the eyes: Billiards knowledge as revealed by gaze shifts in a dynamic visual prediction task. Journal of Vision, vol. 12 (30), pp. 1-19. doi: 10.1167/12.11.30

Duchowski, A.T. (2007). Eye Tracking Methodology: Theory and Practice. Edition: 2nd, Springer.

Egerland, E.M.; Nascimento, J.V. \& Both, J. (2010). Competência profissional percebida de treinadores esportivos catarinenses. Revista da Educação Física, vol. 21 (3), pp. 457-467. doi: 10.4025/reveducfis.v21i3.8285

Furley, P.A. \& Memmert, D. (2015). Creativity and Working Memory Capacity in Sports: Working Memory Capacity Is not a Limiting Factor in Creative Decision Making amongst Skilled Performers. Frontiers in Psychology, vol. 6 (115), pp. 17. doi: 10.3389/fpsyg.2015.00115

Furley, P.A. \& Memmert, D. (2012). Working Memory Capacity as Controlled Attention in Tactical Decision Making. Journal of Sport \& Exercise Psychology, vol. 34, pp. 322-344.

Gil, A.; Moreno, M. P.; García-González, L.; Moreno, A. \& Del Villar, F. (2012). Analysis of declarative and procedural knowledge in volleyball according to the level of pratice and players' age. Perceptual and Motor Skill, vol. 115 (2), pp. 632644. DOI: 10.2466/30.10.25.PMS.115.5.632-644

Gil, A.; Moreno, M.P.; Moreno, A.; García-González, L.; Claver, F. \& Del Villar, F. (2013). Analysis of the Relationship Between the Amount of Training and Cognitive Expertise. A Study of Young Volleyball Players. Journal of Strength \& Conditioning Research, vol. 27 (3), pp.698-702. doi: 10.1519/JSC.0b013e31825d99c9

Gilbert, W.D.; Gilbert, J.N. \& Trudel, P. (2001). Coaching strategies for youth sports. Part 2: personal characteristics, parental influence, and team organization. The Journal of Physical Education, Recreation \& Dance, vol. 72 (5), pp. 41-46. doi: 10.1080/07303084.2001.10605752 
Gorman, A.D.; Abernethy, B. \& Farrow, D. (2011). Investigating the anticipatory nature of pattern perception in sport. Memory \& cognition, vol. 39 (5), pp. 894901. doi: 10.3758/s13421-010-0067-7

Hansen, D.W. \& Hammoud, R.I. (2007). An improved likelihood model for Eye Tracking. Computer Vision and Image Understanding, vol. 106, pp. 220-230. doi: 10.1016/j.cviu.2006.06.012

Hüttermann, S. \& Memmert, D. (2015). The influence of motivational and mood states on visual attention: A quantification of systematic differences and casual changes in subjects' focus of attention. Cognition and Emotion, vol. 29 (3), pp. 471-483. doi: 10.1080/02699931.2014.920767

Hüttermann, S. \& Memmert, D. (2014). Does the inverted-U function disappear in expert athletes? An analysis of the attentional behavior under physical exercise of athletes and non-athletes. Physiology \& Behaviour, vol. 131, pp. 87-92. doi: 10.1016/j.physbeh.2014.04.020

Janelle, C. \& Hatfield, B. (2008). Visual attention and brain processes that underlie expert performance: implications for sport and military psychology.

Military Psychology, vol. 20 (Suppl.1), pp. S39-S69. doi: 10.1080/08995600701804798

Jones, L.; Morgan, K. \& Harris, K. (2012). Developing coaching pedagogy: seeking a better integration of theory and practice. Sport, Education and Society, vol. 15 (2), pp. 1-17. doi: 10.1080/13573322.2011.608936

Kreitz, C.; Furley, P.; Memmert, D. \& Simons, D.J. (2014). Working-memory performance is related to spatial breadth of attention. Psychological Research, vol. 79 (6), pp. 1034-1041. doi: 10.1007/s00426-014-0633-x

Larsson, L.; Nyström, M.; Andersson, R. \& Stridh, M. (2015). Detection of fixations and smooth pursuit movements in high-speed eye-tracking data. Biomedical Signal Processing and Control, vol. 18, pp. 145-152. doi: 10.1016/j.bspc.2014.12.008

Liu, S. (2015). Research on Athletes Visual Search: A Case Study. International Journal of Science and Research, vol. 4 (1), pp. 1373-1376.

Moreno, M.P.; Moreno, A.; Gil, A.; García-Gonzalez, L. \& Del Villar, F. (2016). Influence of experience in procedural knowledge in volleyball: Applications for training. International Journal of Sports Science \& Coaching, vol. 11 (2), pp. 191199. doi: $10.1177 / 1747954116637152$

Nummenmaa, L.; Hyönä, J. \& Calvo, M.G. (2006). Eye movement assessment of selective attentional capture by emotional pictures. Emotion, vol. 6 (2), pp. 257268. doi: 10.1037/1528-3542.6.2.257

Oliveira, R.F.; Lobinger, B.H. \& Raab, M. (2014). An adaptive toolbox approach to the route to expertise in sport. Frontiers in Psychology, vol. 5 (article 709), pp. 1-4. doi: $10.3389 /$ fpsyg.2014.00709

Paquette, K.; Hussain, A.; Trudel, P. \& Camire, M. (2014). Sport federation's attempt to restructure a coach education program using constructivist principles. International Sport Coaching Journal, vol.1 (2), pp. 75-85. doi: 10.1123/iscj.20130006

Piras, A.; Lobietti, R. \& Squatrito, S. (2010). A study of saccadic eye movement dynamic in Volleyball: comparasion between athletes and non-athletes. Journal of Sports Medicine and Physical Fitness, vol. 50 (1), pp. 99-108. 
Piras, A.; Lobietti, R. \& Squatrito, S. (2014). Response Time, Visual Search Strategy, and Anteipatory Skills in Volleyball Players. Journal of Ophthalmology, vol. 2014 (article ID 189268), pp. 1-10. doi: 10.1155/2014/189268

Raab, M. (2015). SMART-ER: a Situation Model of Antecipated Response consequences in Tactical decisions in skill acquisition - Extended and Revised. Frontiers in Psychology, vol. 5 (article 1533), pp.1-5. doi: 10.3389/fpsyg.2014.01533

Raab, M. \& Harwood, C. (2015). Special issue of Psychology of Sport and Exercise "The development of expertise and excellence in sport psychology". Psychology of Sport and Exercise, vol. 16, pp. 1-2.

Raab, M. \& Laborde, S. (2011). When to Blink and When to Think: Preference for Intuitive Decisions Results in Faster and Better Tactical Choices. Research Quarterly for Exercise and Sport, vol. 82 (1), pp.1-10. doi: 10.1080/02701367.2011.10599725

Saez-Gallego, N.M.; Vila-Maldonado, S.; Hernandez, J.A. \& Jordan, O.R.C. (2013). Análisis del comportamiento visual y la toma de decisiones en el bloqueo en Voleibol. Cuadernos de Psicología del Deporte, vol. 13 (2), pp. 31-44.

Sanfey, G.A. (2007). Decision Neuroscience. New directions in studies of judgmentet and decision making. Current Directions Psychological Science, vol. 16 (3), pp. 151-155. doi: 10.1111/j.1467-8721.2007.00494.x

Schempp, P.; Webster, C.; McCullick, B.; Busch, C. \& Mason, I. (2007). How the best get better: an analysis of the self-monitoring strategies used by expert golf instructors. Sport, Education and Society, vol. 12 (2), pp. 175-192. doi: 10.1080/13573320701287502

Schlappi-Lienhard, O. \& Hossner, E-J. (2015). Decision making in beach volleyball defense: Crucial factors derived from interviews with top-level experts. Psychology of Sport and Exercise, vol. 16, pp. 60-73. doi: 10.1016/j.psychsport.2014.07.005

Stokes, M.G.; Atherton, K.; Patai, E.Z. \& Nobre, A.C. (2012). Long-term memory prepares neural activity for perception. Proceedings of the National Academy of Sciences, vol. 109 (6), pp. 360-367. doi: 10.1073/pnas.1108555108

Tenenbaum, G. (2003). Expert Athletes: An integrated Approach to decision making. In: Starkes, J.L. \& Erickson, K.A. (Eds.), Expert Performance in Sports: Advance in Research on Sports Expertise, Champaign: Human Kinetics. pp.192218.

Tien, T.; Pucher, P.H.; Sodergren, M.H.; Sriskandarajah, K.; Yang, G-Z. \& Darzi, A. (2014). Eye Tracking for skills assessment and training: a systematic review. Journal of Surgical Research, vol. 191, pp. 169-178. doi: 10.1016/j.jss.2014.04.032

Vila-Maldonado, S.; Abellan, J.; Saez-Gallego, N.M.; Garcia-Lopez, L.M. \& Contreras, O.R. (2014). Decision-making and visual perception skills in youth volleyball players and non-players. Journal of Sports and Health Research, vol. 6 (3), pp. 265-276.

\section{Número de citas totales / Total references: 46 (100\%) Número de citas próprias de la revista / Journal's own references: $0(0 \%)$}

Rev.int.med.cienc.act.fís.deporte- vol. 19 - número 75 - ISSN: 1577-0354 